\title{
The Catastrophe of Coronal Magnetic Flux Ropes in CMEs
}

\author{
Y. Q. $\mathbf{H u}^{1} \dagger$ \\ ${ }^{1}$ School of Earth and Space Sciences, University of Science and Technology of China, Hefei \\ 230026, China \\ email: huyq@ustc.edu.cn
}

\begin{abstract}
A brief review is given on the progress made in the study of the catastrophe of coronal magnetic flux ropes with implication in coronal mass ejections (CMEs). Relevant studies have been so far limited to 2.5-D cases, with a flux rope levitating in the corona, either parallel to the photosphere in Cartesian geometry or encircling the Sun like a torus in spherical geometry. The equilibrium properties of the system depend on the features of the flux rope and the surrounding background state. Under certain circumstances, the flux rope exhibits a catastrophic behavior, namely, the rope loses equilibrium and erupts upward upon an infinitesimal variation of any control parameter associated with the background state or the flux rope. The magnetic energy of the system right at the catastrophic point may exceed the corresponding open field energy so that after the background field is opened up by the erupting flux rope, a certain amount of magnetic free energy is left for the heating and acceleration of coronal plasma against gravity. The flux rope model has been used to reveal the common features of CMEs and to simulate typical CME events, proving to be a promising mechanism for the initiation of CMEs. Incidentally, the Aly conjecture on the maximum magnetic energy of force-free fields places a serious constraint on 2.5-D flux models. Nevertheless, current sheets must form during a catastrophe on the Alfvén timescale, and magnetic reconnection across the newly formed current sheets may contribute to circumventing such a constraint. In this sense, the catastrophe simply plays a role of driver for the fast magnetic reconnection, and a combination of them is thus responsible for the initiation of CMEs.
\end{abstract}

Keywords. Sun: coronal mass ejections (CMEs), magnetic fields, MHD

\section{Introduction}

Magnetic flux rope, defined as a twisted magnetic loop anchored in the photosphere, is believed to be a typical structure in the solar corona, though there is no direct evidence based on very few and vague magnetic field observations over there. Nevertheless, Yan et al. (2001) found a flux-rope like structure in the corona through a reconstruction of the coronal magnetic field based on the vector magnetograms (figure 1). Theoretically, the existence of such a flux rope serves as a necessary condition for supporting prominences in equilibrium against gravity (Low \& Hundhausen, 1995). Based on observations, prominences have two distinct magnetic configurations, normal and inverse, according to whether their magnetic field is consistent with or opposite to the photospheric magnetic polarity beneath them (Anzer, 1989; Leroy, 1989). Consequently, the associated coronal flux ropes also have two types of magnetic configurations (Zhang \& Low, 2004), but most flux rope models so far belong to the inverse type.

If a flux rope exists in the corona, its eruption must lead to an eruption of the associated prominence below, a coronal mass ejection (CME) above, and possibly, a fast reconnection

$\dagger$ Present address: School of Earth and Space Sciences, University of Science and Technology of China, Hefei 230026, China 


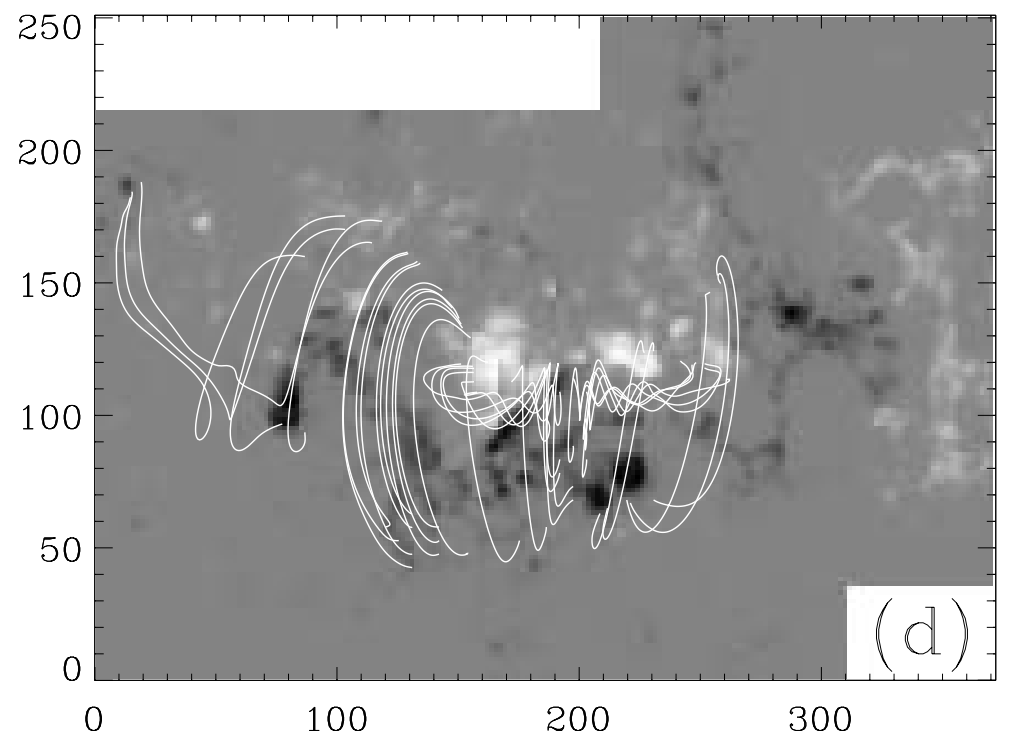

Figure 1. Reconstructed field lines projected on the photospheric magnetogram show clearly a flux rope along the neutral line embraced by overlying arcades (form figure $3 \mathrm{~d}$ of Yan et al., 2001). The tick labels are in the unit of arc seconds.

across the newly-formed vertical current sheet caused by the rope eruption, leading to a two-ribbon solar flare. In terms of this scenario, the magnetic flux rope is naturally and organically related to various solar explosive phenomena. On the other hand, the eruption of the flux rope is caused by a change of either the background state or the rope itself, and such a change is believed to be created by photospheric activities such as random motions, magnetic emergence and cancellation, and magnetic reconnection, which are generally slow compared to explosive events. Therefore, the rope eruption should take a catastrophic manner, namely, when the flux rope system evolves to a certain critical state, an infinitesimal perturbation will cause a sudden transition of the system from equilibrium into a dynamic state: the flux rope suddenly loses equilibrium and erupts upward at a fraction of the local Alfvén speed.

Both analytical studies and numerical simulations have been made to explore the equilibrium properties and catastrophic behaviors of coronal flux ropes in order to find a physically sound mechanism for solar explosive phenomena. We will review various flux rope models with emphasis on the catastrophic conditions in section 2 . The catastrophic energy threshold and its relation to the Aly conjecture is discussed in section 3. We conclude with the implication of the flux rope catastrophe in CMEs in section 4.

\section{MHD Coronal Flux Rope Models}

To our knowledge, the earliest flux rope model is attributed to Van Tend \& Kuperus (1978) who approximated the flux rope by a wire current filament (figure 2a) and concluded that a loss of equilibrium occurs if the current in the filament exceeds a critical value. However, in their model and subsequent similar ones, the field of the wire filament and the background field are freely reconnected, so the ideal magnetohydrodynamic (MHD) condition is disregarded. Soon their simple wire filament model was refined and replaced by the so-called thin-rope model, in which the ideal MHD condition is taken into account and thus electric current sheets appear in the solution (figure $2 \mathrm{~b}$ ). 

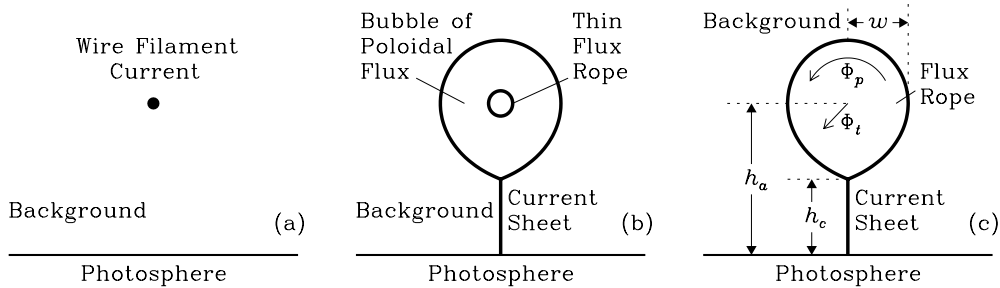

Figure 2. Schematic representation of coronal flux rope models: (a) wire filament current, (b) thin flux rope with a bubble, and (c) flux rope of finite cross section without bubble.

The flux rope is thin in the sense that its radius is far smaller than the length scale of the photospheric field, an approximation purely for analytical tractability. The thin-rope model was then extended to numerical rope models, where the rope is finite in radius (figure 2c).

\subsection{Description of the Flux Rope System}

Before moving on to discuss different types of flux rope models, it is opportune at this point to describe the fundamental features of the flux rope system and introduce several parameters which control and characterize the flux rope.

As shown schematically in figure 2c, the system consists of two topologically disconnected regions, a flux rope and a surrounding background state. The rope is characterized by its annular magnetic flux $\Phi_{p}$ per length in Cartesian geometry or per radian in spherical geometry, and axial flux $\Phi_{t}$. If the gravity is incorporated, then the total mass $M$ inside the rope becomes another crucial parameter. In addition, the following parameters may be introduced to represent the geometrical features of the flux rope: the height $h_{a}$ of the rope axis, the length $h_{c}$ of the vertical current sheet formed as the rope is detached from the photosphere, and the half-width $w$ of the rope. For thin-rope models, the axial flux is limited inside a rope of circular section, which is surrounded by a "bubble" of purely poloidal flux (figure $2 \mathrm{~b}$ ). As a result, $\Phi_{t}$ is replaced by the ratio between the current intensity of the rope and the force-free factor (Lin et al., 1998), $w$ by the radius of the rope, and $\Phi_{p}$ by the poloidal flux in the bubble. For numerical models, the field in the bubble is also twisted so that, as the term suggests, the flux rope comes in contact with the background field, needless to introduce an additional bubble between them.

There exist various choices for the background field. A simplest one is a closed bipolar potential field, which was mostly adopted in thin-rope models. Closed multipolar potential field was also used; it gives a more dynamic evolution or jump of the flux rope than the bipolar field does. For numerical solutions, one may have more choices, for instance, a partly-open potential field with an equatorial current sheet, a quadrupolar field with a neutral point in the corona, or a helmet streamer with several bipolar fields inside. The background field may be changed by photospheric motions, magnetic reconnections, magnetic emergence and cancellation, or simply by a variation of the source strength. Moreover, a more realistic choice is a partly-open magnetic field with a quasimagnetostatic helmet streamer in the closed field region and a steady solar wind outside.

Theoretically, one may introduce a set of parameters that control the physical properties of the background state and the flux rope. By solving the equilibrium equations of the system, we may determine the variation of the geometrical parameters caused by a change in the control parameters. If the variation is discontinuous at a certain point, then a catastrophe occurs for the system when the control parameters cross that point. For simplicity, we may let one of the control parameters changeable, denoted by $\lambda$, while keeping others fixed. On this basis, each geometrical parameter, denoted by $h$, can be 

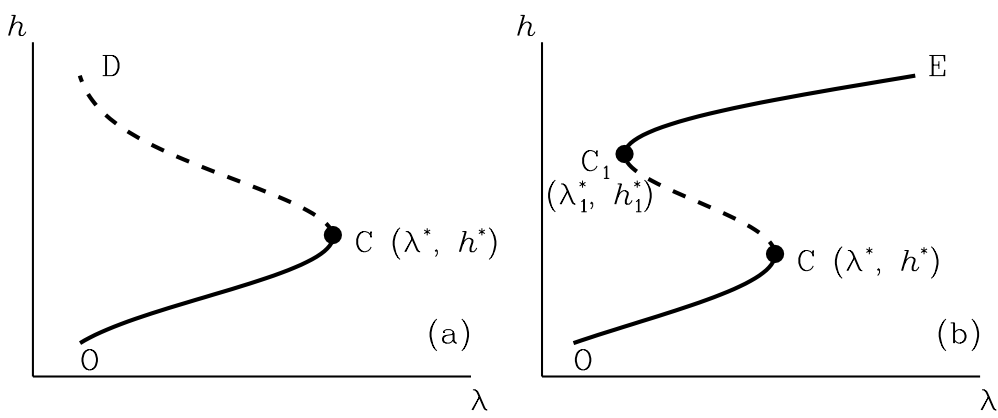

Figure 3. Schematic representation of equilibrium curves associated with a catastrophe of a system. The solid curves represent stable equilibrium states of the system whereas the dashed unstable ones. $\mathrm{C}$ and $\mathrm{C}_{1}$ are the nose points at which a catastrophe takes place.

determined as a function of the single control parameter $\lambda$. Figure 3 shows schematically two typical $h-\lambda$ profiles associated with a catastrophe, where the solid curve represents stable equilibrium states and the dashed unstable equilibrium states. On the fold-shaped profile OD in figure 3a, there is one nose point $\mathrm{C}$, at which $\lambda=\lambda^{*}$ and no equilibrium is available beyond for the system. For $\lambda<\lambda^{*}$, there exist two equilibrium solutions, one stable and the other unstable. As $\lambda$ increases beyond $\lambda^{*}$, the system loses equilibrium, and $h$ approaches to infinity. In figure $3 \mathrm{~b}$, the profile $\mathrm{OE}$ is of S-type, and it has two nose points, $\mathrm{C}$ and $\mathrm{C}_{1}$, with $\lambda=\lambda^{*}$ and $\lambda_{1}^{*}$ respectively. For $\lambda_{1}^{*}<\lambda<\lambda^{*}$, there are three equilibrium solutions, one unstable and the other two stable. Across both $\mathrm{C}$ and $\mathrm{C}_{1}$, a catastrophe occurs, and $h$ jumps up and down respectively with a finite amplitude, namely, the system loses its original equilibrium and reaches a new one. The catastrophe associated with the nose point $\mathrm{C}$ is more relevant to our purpose, since we are interested in the eruption and expansion of the system with a positive jump of $h$.

\subsection{Catastrophe Associated with the Rope Property Change}

As mentioned above, the rope system evolves with the change of the rope properties, including the annular flux $\Phi_{p}$, the axial flux $\Phi_{t}$, and the total mass $M$ within the rope. For thin-rope models, the gravity is ignored, $\Phi_{t}$ is usually fixed, and $\Phi_{p}$, identified as the poloidal flux in the surrounding bubble, is to be adjusted. In the thin-rope model proposed by Forbes \& Isenberg (1991) in Cartesian geometry, the background field is produced by a line dipole at a depth $d$ below the photosphere, and a flux rope of radius $r \ll d$ is embedded in the bubble. A magnetic reconnection at the photosphere right below the flux rope leads to a gradual increase of $\Phi_{p}$ in the bubble. It was found that for $r / d<10^{-3}$, the rope system exhibits a catastrophic behavior in relation to $\Phi_{p}$. The rope loses equilibrium at the catastrophic point and enters a new one with a vertical current sheet below. By taking a background field created by a submerged line quadrupole instead, Isenberg et al. (1993) found a similar catastrophe for $r / d<0.23$.

A 2.5-D, time-dependent ideal MHD model in Cartesian coordinates was used to find equilibrium solutions associated with a coronal flux rope of large cross section embedded in a background field ( $\mathrm{Hu} \& \mathrm{Liu}, 2000 ; \mathrm{Hu}, 2001,2002 ; \mathrm{Hu} \&$ Jiang, 2001; Li \& Hu, 2001, 2003; Wang \& Hu, 2003). It was demonstrated that the catastrophic behavior of the system depends crucially on the pattern of the background magnetic field. For a completely closed background field, catastrophe does not exist no matter how the background field is produced, say, by two separated extended sources of opposite sign (Hu \& Liu, 2000), or by a line dipole, quadrupole, or octapole at a depth below the photosphere (Wang \& $\mathrm{Hu}, 2003$ ). This result is consistent with the above-mentioned 
conclusion reached by thin-rope models that no catastrophe occurs for flux ropes of large radius. However, there exists a steep segment in the profiles of the geometric parameters versus $\Phi_{p}$ or $\Phi_{t}$, and the faster the background field decays with height, the larger both the gradient and the growth amplitude within the segment will be (Wang \& $\mathrm{Hu}, 2003$ ).

On the other hand, a flux rope underneath a partly-open background field exhibits a catastrophic behavior during the slow change of the magnetic fluxes of the rope, namely, there exists a certain point, at which an infinitesimal enhancement of these fluxes causes a jump of the geometrical parameters of the rope $(\mathrm{Hu}, 2001,2002)$. In addition, the amplitude of the jump depends on the extent to which the background magnetic field is open, approaching infinity for fully-open background field ( $\mathrm{Li} \& \mathrm{Hu}, 2001,2002$ ), and zero for closed one. Incidentally, a drainage of mass from the rope may also lead to the expansion of the rope and thus a catastrophe of the system, as expected and demonstrated by tentative simulations.

In an extension of the flux rope model to spherical geometry, $\mathrm{Hu}$ et al. (2003) found that a flux rope embedded in a bipolar background field also exhibits a catastrophic behavior with respect to $\Phi_{p}$ or $\Phi_{t}$, which does not depend on whether the background field is partly-open or closed. The rope sticks to the solar surface in equilibrium below the catastrophic point, and escapes to infinity above. The catastrophic amplitude is infinite in this case. However, under some special circumstances when a downward force on the rope dominates, a flux rope in spherical geometry may also levitate stably with a vertical current sheet below in the frame of ideal MHD, and catastrophe may either be non-existent or finite in amplitude. For instance, in a quadrupolar background field with a neutral point in the corona between the central and ambient bipolar fields, a flux rope underneath the central bipolar field may lead to the formation of a transverse current sheet right above the flux rope with a current opposite to the rope current. The two currents are repellent so that the rope is subject to a downward force (Zhang et al., 2004). Besides, the gravitational force on the rope is also downward, whatever the background field is. In the analytical solutions of magnetic flux ropes of both inverse and normal configurations obtained by Zhang \& Low (2004), the gravity associated with the total mass inside the rope is crucial to hold the system in equilibrium especially for ropes of normal configuration. If these downward forces happen to dominate, the flux rope may levitate stably high in the corona, and a catastrophe, if any, must be finite in amplitude, or even no catastrophe occurs for the system. This issue is left for future numerical studies.

\subsection{Catastrophe Associated with Photospheric Motions}

The background field is subject to change under the action of photospheric motions, which have a subtle influence on the behavior of the flux rope. In terms of a thin-rope model, Forbes \& Priest (1995) found that the converging motion of the two magnetic sources of opposite polarity leads to a catastrophe of the rope if the radius of the rope is smaller than a certain critical value. Lin et al. (2002) studied the evolution of a semicircular flux rope with two ends anchored in the photosphere with varying strength and distance of the background field sources, and concluded that the evolution due to the change in source strength shows the likelihood of catastrophic loss of equilibrium. In a model developed by $\mathrm{Hu} \&$ Jiang (2001) and $\mathrm{Hu}$ (2002), the two magnetic fluxes of the rope, $\Phi_{p}$ and $\Phi_{t}$, were fixed, and the background field was partly open, produced by two separated magnetic sources of opposite sign at the photosphere and changed by three types of photospheric motions: a reduction of the interval between the two sources, a contraction of each source, and a shear of the closed part of the field respectively. The system exhibits a catastrophe such that there exists a certain critical point for each type 
of photospheric motions, across which an infinitesimal displacement on the photosphere causes a finite jump of the geometrical parameters of the rope.

\subsection{Catastrophe with Both Background Field and Rope Properties Changed}

There were flux rope models in which both the background field and the flux rope are changed in property. For instance, Isenberg et al. (1993) let the background field decrease with time in strength and the decreased flux transfer to the bubble around the rope. As such, they found a catastrophe with respect to the background field strength. The same approach was taken by Lin et al. (1998) but for a dipole background field and a torus-shaped flux rope in spherical geometry, leading to a similar conclusion. In these models the flux transfer between the bubble and background is implemented via a reconnection at the photosphere right below the rope. A reconnection across the newly formed current sheet in the corona during the rope ascending might be also invoked to achieve the transfer, provided that the evolution of the system is sufficiently slow. Lin \& van Ballegooijen (2002) assumed that the vertical current sheet appearing in the thin-rope model obtained by Forbes \& Isenberg (1991) is suppressed by magnetic reconnection and replaced by an X-type neutral point, and found that the new configuration exhibits a catastrophic behavior that is no longer constrained by the radius of the rope. In the study of the interaction between an existing flux rope system and a new emerging flux, Lin et al. (2001) allowed the background field, the field in the bubble around the rope, and the new emerging field to reconnect freely such that the resultant field is potential everywhere except in the thin flux rope. As a result, no current sheet appears in the solution. Then, in terms of the profiles of the positions of the flux rope axis versus various control parameters, they found quite a lot of cases with catastrophe.

\subsection{Flux Rope Catastrophe in a Background Solar Wind}

Most flux rope models available in literature assumed that the background coronal plasma is in magnetostatic equilibrium. However, solar wind does exist and certainly exerts a critical influence on the flux rope equilibrium and the associated catastrophe. Wu et al. (1997a) presented a numerical model with a helmet streamer surrounded by a steady solar wind and a flux rope embedded in the streamer. They found that the flux rope either sticks to the solar surface in equilibrium or erupts upward depending on the magnetic energy level of the system. For the former, a photospheric shear motion applied to the streamer base (Wu \& Guo, 1997a) or an enhancement of the axial flux $\left(\Phi_{t}\right)$ of the rope (Wu \& Guo, 1997b) destabilizes the system, leading to an eruption of the rope. This implies an existence of the catastrophe of the flux rope in the solar wind background, though the catastrophic point was not identified yet. Sun \& Hu (2004) used a similar model to $\mathrm{Wu}$ and Guo's and found the catastrophic point with respect to the variation of $\Phi_{t}, \Phi_{p}$, and $M$, respectively. In comparison with magnetostatic models in the absence of solar wind, the coronal plasma helps open up the external part of the helmet streamer that is expanding with the increase of magnetic fluxes of or the drainage of mass from the flux rope. This reduces the tension force of the helmet streamer on the flux rope and makes it easier for the catastrophe to occur.

\section{Catastrophic Energy Threshold of the Flux Rope System}

An important issue in the study of flux rope catastrophe is the magnetic energy threshold, referred to as $W_{c}$ hereafter, across which a catastrophe takes place. One hopes that $W_{c}$ would exceed the energy of the corresponding open field, referred to as $W_{\text {open }}$, so that after the field is opened up by a catastrophe, there is a certain amount of magnetic 
free energy left for the heating and acceleration of coronal plasma. However, such an expectation is faced with a serious challenge since Aly (1984) put forward a conjecture saying that in an infinite domain and for a given distribution of normal field at the lower boundary, the maximum energy of force-free fields with at least one end of each field line anchored in the lower boundary is $W_{\text {open }}$. The Aly conjecture was supported by numerical (Yang et al., 1986; Mikić \& Linker, 1994; Roumeliotis et al., 1994; Amari et al., 1996) and analytical (Lynden-Bell \& Boily, 1994; Aly, 1994; Wolfson, 1995) examples. Meanwhile, Aly (1991) and Sturrock (1991) addressed proofs of the conjecture, respectively, based on some intuitive assumptions. Recently, the Aly conjecture was extended by $\mathrm{Hu}$ (2004) in such a way that it is impossible to store more magnetic energy in the corona by photospheric shear motions at the base of any part of the closed flux of a force-free field than that of the field in which the sheared closed flux opens but the rest remains closed. Several authors argued that counter-evidence was found to deny the Aly conjecture (e.g., Choe \& Cheng, 2002; Wolfson \& Low, 1992). However, these authors took a boundary condition at infinity, which forced all allowable force-free fields to be completely closed and might thus change the nature of the solutions in a dramatic way $(\mathrm{Hu}, 2004)$, so their conclusions seem to be questionable.

Under 2-D approximations, one has to select a spherical geometry so as to make a physically reasonable analysis in energetics. For force-free fields in the exterior of a sphere of radius $R_{0}$ with a given radial field at the sphere, the magnetic energy satisfies the following inequality (Aly, 1984)

$$
W_{p} \leqslant W \leqslant\left. W_{\max } \equiv \frac{R_{0}^{3}}{2 \mu} \int_{0}^{2 \pi} d \phi \int_{0}^{\pi} B_{r}^{2}\right|_{r=R_{0}} \sin \theta d \theta,
$$

where $W_{p}$ is the energy of the corresponding potential field. Therefore, we have $W_{\text {open }} \leqslant$ $W_{\max }$. For 2-D flux rope configurations, there exist field lines which are detached from the solar surface, so the force-free field energy may exceed $W_{\text {open }}$ (Priest \& Forbes, 1990). The percentage of this energy in excess of $W_{\text {open }}$ has an upper bound $\left(W_{\max } / W_{\text {open }}-1\right)$. Taking the dipole field as an example, we have $W_{p}=4 \pi B_{0}^{2} R_{0}^{3} /(3 \mu)$ (where $B_{0}$ is the field strength at the equator and $R_{0}$ the solar radius), $W_{\text {open }}=1.662 W_{p}$ (Low \& Smith, 1993; Mikić \& Linker, 1994), and $W_{\max }=2 W_{p}$, so the maximum energy in excess of $W_{\text {open }}$ is $20.3 \%$.

In terms of a 2.5-D flux rope model in spherical coordinates, $\mathrm{Hu}$ et al. (2003) concluded that $W_{c}$ is slightly larger than $W_{\text {open }}$, and the gravity associated with the prominence supported by the flux rope raises $W_{c}$ by an amount that is approximately equal to the magnitude of the excess gravitational energy associated with the prominence. Such a conclusion was further quantified by $\mathrm{Li} \& \mathrm{Hu}(2003): W_{c}$ exceeds $W_{\text {open }}$ by $8 \%$. Flyer et al. (2004) found a 2-D force-free field solution with detached field lines which should be below the catastrophic point but has an energy larger than $W_{\text {open }}$ by $3.5 \%$. Using a cold plasma approximation, Zhang \& Low (1004) found magnetostatic equilibrium solutions of both inverse and normal prominence fields which have magnetic energy larger than $W_{\text {open. }}$. The flux rope is kept in equilibrium by gravity, and more mass is needed for trapping flux ropes of normal type. Therefore, a drainage of plasma out of a prominence will lead to a rope eruption.

In the presence of a background solar wind, $W_{c}$ may be also larger than $W_{\text {open }}$. Using a polytropic solar wind model, Guo \& Wu (1998) found quasi-static helmet streamer solutions that contain a flux rope with or without cavity, and calculated the magnetic energy in the computational domain (1-6 $\left.R_{0}\right)$. The open field energy in the same domain was also calculated for comparison. They found that the solution associated with a cavity flux rope has more energy than the open field. Sun \& Hu (2004) used a similar model 
to study the catastrophe of the flux rope system and to determine the value of $W_{c}$. The threshold was found to be larger than $W_{\text {open }}$ by about $8 \%$ that is the same as obtained for magnetostatic equilibrium solutions ( $\mathrm{Li} \& \mathrm{Hu}, 2003)$. Also, with increasing mass inside the flux rope, $W_{c}$ increases by an amount that is approximately equal to the magnitude of the excess gravitational energy associated with the enhanced mass in the flux rope (Sun \& Hu, 2004).

In general, a realistic coronal flux rope should have its ends anchored in the photosphere. If we believe that the Aly conjecture is correct, a system with a flux rope anchored in the photosphere can never have an energy in excess of $W_{\text {open }}$. If a catastrophe exists for such a system, $W_{c}$ must be smaller than $W_{\text {open }}$, the rope ascends and expands after catastrophe, and the background field around the rope remains to be invariant in topology. To make the catastrophe develop into a plausible eruption, one has to invoke magnetic reconnection across the newly formed current sheet below the ascending rope, which leads to a transfer of magnetic flux from the background field into the rope and the newly formed helmet arcade below the rope. $W_{c}$ needs only to be larger than the energy of the corresponding partly-open field instead of $W_{\text {open }}$. It is the catastrophe that creates a current sheet at the Alfvén timescale as an ideal MHD process and provides a favorable site for fast magnetic reconnection. A combination of the catastrophe and the follow-up fast reconnection is thus responsible for the rope eruption and the initiation of CMEs. On the other hand, if the Aly conjecture becomes invalid for magnetic configurations with a catastrophic behavior, $W_{c}$ may be still larger than $W_{\text {open }}$, as inferred by Li \& $\mathrm{Hu}$ (2003). It is interesting to check such a possibility by 3-D numerical calculations.

\section{Flux Rope Catastrophe and CMEs}

The coronal flux rope structure and its catastrophe were used to explain the observed features of CMEs, mostly in qualitative levels. As mentioned above, present flux rope models have been limited to 2-D cases in either Cartesian or spherical geometry. The two types of models differ from each other while applied to CMEs.

For models in 2-D Cartesian geometry it is energetically impossible for the flux rope to open the background field in the frame of ideal MHD. When a catastrophe occurs, the flux rope jumps at the Alfvén timescale but by a finite height, so the catastrophe plays a trigger of CMEs at most. In order for a real eruption of the rope, one has to rely on a magnetic reconnection across the newly formed current sheet below the rope. The motion pattern is determined entirely by the reconnection rate, so it is of no importance whether a catastrophe really takes place. In the absence of catastrophe, Lin \& van Ballegooijen (2002) concluded that a slow reconnection at the photosphere leads to a continuous upward motion of the flux rope, which may account for slow CMEs. On the other hand, Lin \& Forbes (2000), Lin (2002), and Lin et al. (2004) introduced a reconnection somewhere in the middle of the current sheet, allowing the system to evolve into a configuration consisting of an expanding helmet arcade below and an ascending bubble containing the rope above, connected by the vertical current sheet. For a given constant reconnection rate $M_{A}$, the Alfvén number of the inflow into the reconnection site, the motion patterns of these features are determined by the coronal Alfvén speed and its variation with height. Taking $M_{A}=0.1$ and a realistic density profile with height, these authors explained the peculiar motion of giant X-ray arches and anomalous postflare loops and the observed features of three-component (bright dome, dark cavity, and dense core) CMEs at heights of a few solar radii.

On the other hand, the flux rope may erupt to infinity after catastrophe in 2-D spherical geometry, at least for a bipolar background field. Therefore, by itself, a catastrophe is 
sufficient to implement the eruption process associated with CMEs. Hu et al. (2003) took an isothermal static corona as the background and obtained profiles of the geometrical parameters of the rope with height after catastrophe. The flux rope erupts at the Alfvén timescale that increases with height, leading to an initial sharp acceleration followed by a gradual deceleration. The eventual speed of the rope axis is less than $100 \mathrm{~km} / \mathrm{s}$. However, if the effects of the solar wind and the magnetic reconnection are considered, the motion pattern of the erupting rope will be different. In a solar wind background, the speed of the flux rope axis approaches the solar wind speed at large distance from the Sun (Wu et al., 1997a). Wu et al. (1997b, 1999) applied their flux rope model in the presence of solar wind to simulate two CME events observed by the Large-Angle and Spectrometric Coronagraph Experiment (LASCO) in July 1996 and January 1997. The eruption was presumed to be caused by an enhancement of $\Phi_{t}$ (azimuthal flux) of the rope for the second event, and a simultaneous increase of $\Phi_{t}$ and decrease of $M$ (mass) of the rope for the first. The simulation results were compared with LASCO observations in the near Sun region and Wind observations at $1 \mathrm{AU}$, showing a reasonable agreement in shape with LASCO images and a qualitative resemblance to Wind observations. Note that in the model by Sun \& Hu (2004), a special measure is taken to completely suppress any reconnection across the vertical current sheet below the rope, whereas in Wu et al.'s model, a numerical reconnection exists across the sheet and a helmet arcade forms during the eruption of the rope. The numerical reconnection helps the rope erupt, but ignores the Joule heating that would have occurred and caused a coronal heating and a further acceleration of the rope eruption.

So far we were limited to the flux rope of inverse type. Based on a qualitative analysis, Low \& Zhang (2002) and Zhang \& Low (2004) argued that flux ropes of normal type are apt to produce fast CMEs in which magnetic reconnection plays a crucial role, whereas the expulsion of flux ropes of inverse type seems likely to involve a gradual acceleration without magnetic reconnection necessarily playing a principle role. Some observational evidence was found in support of such an argument (Zhang et al., 2002).

Although the available flux rope catastrophe models are still too simplified and idealized to really explain the exact triggering mechanisms and quantitative dynamical behaviors of CMEs, they may after all be accepted as a promising mechanism at least for the initiation of three-component CMEs. Further work needs to be done to refine these models so as to examine the interplay between ideal and nonideal MHD processes and to consider three-dimensional effects. configurations.

\section{Acknowledgements}

This work was supported by grants NNSFC 40274049 and 10233050, and grant NKBRSF G2000078404 in China.

\section{References}

Aly, J. J. 1984, ApJ 283, 349

Aly, J. J. 1991, ApJ 375, L61

Aly, J. J. 1994, A\&BA 288, 1012

Amari, T., Luciani, J. F., Aly, J. J., \& Tagger M. 1996, ApJ 466, L39

Anzer, U. 1989, in: E. R. Priest (ed.), Dynamics and Structure of Quiescent Solar Prominences, (Kluwer Academic Publishers, Dordrecht, Holland), p. 143

Choe, G. S. \& Cheng, C. Z. 2002, ApJ (Letters) 574, L179

Flyer, N., Fornberg, B., Thomas, S., \& Low, B. C. 2004, ApJ 606, 1210

Forbes, T. G. \& Isenberg, P. A. 1991, ApJ 373, 294

Forbes, T. G. \& Priest, E. R. 1995, ApJ 446, 377 
Guo, W. P. \& Wu, S. T. 1998, ApJ 494, 419

Hu, Y. Q. 2001, Solar Phys. 200, 115

Hu, Y. Q. 2002, in: H. N. Wang \& R. L. Xu (eds.), Solar-Terrestrial Magnetic Activity and Space

Environment, COSPAR Colloquia Series (Pergamon), Vol. 14, p. 117

Hu, Y. Q. 2004, ApJ 607, 1032

Hu, Y. Q. \& Jiang, Y. W. 2001, Solar Phys. 203, 309

Hu, Y. Q. \& Liu, W. 2000, ApJ 540, 1119

Hu, Y. Q., Li, G. Q., \& Xing, X. Y. 2003, J. Geophys. Res. 108(A2), 1072, doi:10.1029/2002JA009419

Isenberg, P. A., Forbes, T. G., \& Démoulin, P. 1993, ApJ 417, 368

Leroy, J. L. 1989, in: E. R. Priest (ed.), Dynamics and Structure of Quiescent Solar Prominences (Kluwer Academic Publishers, Dordrecht, Holland), p. 77

Li, G. Q. \& Hu, Y. Q. 2001, Chinese Science (Series A (Supplement) 31, 53

Li, G. Q. \& Hu, Y. Q. 2003, Chin. J. Astron. Astrophys. 3, 555

Lin, J. 2002, Chin. J. Astron. Astrophys. 2, 539

Lin, J. \& Forbes, T. G. 2000, J. Geophys. Res. 105, 2375

Lin, J. \& van Ballegooijen, A. A. 2002, ApJ 576, 485

Lin, J., Forbes, T. G., \& Démoulin, P. 1998, ApJ 504, 1006

Lin, J., Forbes, T. G., \& Isenberg, P. A. 2001, J. Geophys. Res. 106, 25053

Lin, J., Raymond, J. C., \& van Ballegooijen, A. A. 2004, ApJ 602, 422

Lin, J., van Ballegooijen, A. A., \& Forbes, T. G. 2002, J. Geophys. Res. 107, 1438

Low, B. C. \& Hundhausen, J. R. 1995, ApJ 443, 818

Low, B. C. \& Smith, D. F. 1993, ApJ 410, 412

Low, B. C. \& Zhang, M. 2002, ApJ 564, L53

Lynden-Bell, D. \& Boily C. 1994, MNRAS 267, 146

Mikić, Z. \& Linker, J. A. 1994, ApJ 430, 898

Priest, E. R. \& Forbes, T. G. 1990, Solar Phys. 126, 319.

Roumeliotis, G., Sturrock, P. A., \& Antiochos, S. K. 1994, ApJ 423, 847

Sturrock, P. A. 1991, ApJ 380, 655

Sun, S. J. \& Hu, Y. Q. 2004, Coronal flux rope catastrophe in a background solar wind, to be submitted.

Van Tend, W. \& Kuperus, M. 1978, Solar Phys. 59, 115

Wang, Z. \& Hu, Y. Q. 2003, Chin. J. Astron. Astrophys. 3, 241

Wolfson, R. 1995, ApJ 443, 810

Wolfson, R. \& Low, B. C. 1992, ApJ 391, 353

Wu, S. T. \& Guo, W. P. 1997a, Adv. Space Sci. 20, 2313

Wu, S. T. \& Guo, W. P. 1997b, in: N. Crooker, J. Joselyn \& J. Feynman (eds.), Coronal Mass Ejections, Geophysical Monograph 99 (AGU, Washington, D. C.), p. 83

Wu, S. T., Guo, W. P., \& Dryer, M. 1997a, Solar Phys. 170, 265

Wu, S. T., Guo, W. P., Andrews, M. D., et al. 1997b, Solar Phys. 175, 719

Wu, S. T., Guo, W. P., Michels, D. J., \& Burlaga, L. F. 1999, J. Geophys. Res. 104, 14789

Yan, Y. H., Deng, Y. Y., Karlicky, M., et al. 2001, ApJ (Letters) 551, L115

Yang, W. H., Sturrock, P. A., \& Antiochos, S. K. 1986, ApJ 309, 383

Zhang, Y. Z., Hu, Y. Q., \& Wang, J. X. 2004, in this proceeding

Zhang, M. \& Low, B. C. 2004, ApJ 600, 1043

Zhang, M., Golub, L., Deluca, E., \& Burkepile, J. 2002, ApJ 574, L97

\section{Discussion}

Kouтснмy: As an observer, I would like to better understand what would be a good candidate for seeing a rising flux rope which eventually drives an eruption or a flare. Is it a filament or is it the cavity which is around a filament, or something else?

Hu: Theoretically, the filament is situated right below the axis of a flux rope, so the cavity around the filament might be a good candidate for either static or rising flux rope. 
Zhukov: The Aly-sturrock conjecture (or theorem) deals with force-free fields. Why do you mention it in connection with CMEs, when the magnetic force is almost certainly playing a role and the field is thus not force-free?

Hu: There are two issues in connection with CMEs, one about the energetics and the other the dynamical process. Force-free field is a good approximation for the first issue, whereas the magnetic force plays a crucial role for the second, and thus the field cannot be considered as force-free.

ForBes: Comment: I agree with your remark that the Aly-Sturrock conjecture has not been rigorously proved for all possible configurations. However, even if it is true, it is also possible to get an eruption by just partly opening the field.

Hu: Yes, but then non-ideal MHD effect such as magnetic reconnection must be invoked in order to make part of the field lines close back to the photosphere. 